\title{
Correction to: Molecular dissection of sugar related traits and it's attributes in Saccharum spp. hybrids
}

\author{
Md. Sariful Islam • Xiping Yang • Sushma Sood • Jack C. Comstock • \\ Fenggang Zan $\cdot$ Jianping Wang
}

Published online: 24 October 2018

(C) Springer Nature B.V. 2018

Correction to: Euphytica (2018) 214:170

https://doi.org/10.1007/s10681-018-2252-x

This article has mistakenly been processed as Review Article, whereas it should have been published as Original Article and should be regarded as such by the reader.

The original article can be found online at https:// doi.org/10.1007/s10681-018-2252-x.

Md. S. Islam $(\bowtie) \cdot S$. Sood · J. C. Comstock

Sugarcane Production Research Unit, USDA ARS,

Canal Point, FL 33438, USA

e-mail: Md.Islam@ARS.USDA.GOV

X. Yang $\cdot$ J. Wang

Agronomy Department, University of Florida,

Gainesville, FL 32610, USA

F. Zan

Sugarcane Research Institute, Yunnan Academy of

Agricultural Sciences, Kaiyuan 661699, Yunan Province,

China 